\title{
Construção e Análise das Propriedades Psicométricas da Escala de Envolvimento Parental na Decisão Vocacional (EEPDV)
}

\author{
Cássia Ferrazza Alves ${ }^{1}$, Luciana Rubensan Ourique Masiero, Ana Cristina Garcia Dias, Marco Antônio Pereira Teixeira \\ Universidade Federal do Rio Grande do Sul, Porto Alegre-RS, Brasil
}

\section{RESUMO}

O envolvimento dos pais durante o processo de decisão profissional dos filhos tem sido considerado uma variável de apoio importante. Este estudo visa apresentar as etapas de construção e as propriedades psicométricas da Escala de Envolvimento Parental na Decisão Vocacional. Esta foi elaborada para avaliar a percepção dos adolescentes sobre as atitudes dos pais diante do processo de escolha profissional. Participaram 390 jovens com idades entre 14 e 20 anos (51,8\% do sexo masculino). A partir das análises fatoriais exploratórias, foram extraídos dois fatores (Apoio à tomada de decisão vocacional e Apoio à exploração vocacional), obtendo-se bons valores de consistência interna $(\alpha=0,76$ a $\alpha=0,85)$. As análises permitiram identificar uma estrutura fatorial adequada e evidências de fidedignidade, indicando que a escala possui potencial para ser utilizada no âmbito da pesquisa e da prática de aconselhamento de carreira.

Palavras-chave: desenvolvimento de carreira; relações familiares; escolha ocupacional; desenvolvimento do adolescente; construção de teste.

\section{ABSTRACT - Construction and Analysis of Psychometric Properties of the Parental Involvement in Vocational} Decision Scale (EEPDV)

Parent's involvement during children's professional decision-making process has been considered a significant support variable. This study aims to present the construction steps and psychometric properties of the Parental Involvement in Vocational Decision Scale. The scale was elaborated to evaluate adolescents' perception of their parents' attitudes towards the professional choice process. A total of 390 youth between 14 and 20 years of age ( $51.8 \%$ males) participated. Two factors were extracted from the exploratory factor analysis (support for vocational exploration and support for vocational decision-making), obtaining good internal consistency values $(\alpha=0.76$ a $\alpha=0.85)$. The analyses permitted identification of an adequate factorial structure and reliability evidence, indicating that the scale has potential to be used in the scope of the research and practice of career counseling.

Keywords: career development; family relationships; occupational choice; adolescent development; test construction.

RESUMEN - Construcción y Análisis de las Propiedades Psicométricas de la Escala de Participación Parental en la Decisión Vocacional (EEPDV)

La participación de los padres durante el proceso de decisión profesional de los hijos ha sido considerada una variable de apoyo importante. Este estudio busca presentar las etapas de construcción y las propiedades psicométricas de la Escala de Participación Familiar en la Decisión Vocacional. Este fue desarrollado para evaluar la percepción de los adolescentes sobre las actitudes de los padres frente el proceso de elección profesional. Participaron 390 jóvenes con edades comprendidas entre 14 y 20 años (51,8\% del sexo masculino). A partir de los análisis factoriales exploratorios, se extrajeron dos factores (Apoyo a la toma de decisión vocacional y Apoyo a la exploración vocacional), obteniéndose buenos valores de consistencia interna $(\alpha=0,76$ a $\alpha=0,85)$. Los análisis permitieron identificar una estructura factorial adecuada y evidencias de fiabilidad, indicando que la escala posee el potencial para ser utilizada en el ámbito de la investigación y la práctica de la orientación profesional.

Palabras clave: desarrollo de carrera; relaciones familiares; elección profesional; desarrollo del adolescente; construcción de test.

O envolvimento parental tem sido evidenciado na literatura como importante para o desenvolvimento dos filhos e, no que tange aos processos de tomada de decisão profissional destes, tem sido destacado na literatura como uma importante influência nas decisões de carreira (Araújo et al., 2012; Bryant, Zvonkovic, \& Reynolds,
2006; Garcia, Restubog, Bordia, Bordia, \& Roxas, 2015). Por exemplo, pais que acreditam nas habilidades dos filhos e demonstram interesse pelas atividades que eles gostam contribuem tanto para a sua independência e autonomia como para a construção de planos para o futuro (Araújo et al., 2012; Keller \& Whiston, 2008). Pais podem 
auxiliar o adolescente a explorar crenças e valores ocupacionais, a desenvolver comportamentos relacionados à exploração de interesses vocacionais e a fortalecer aspirações acadêmicas e ocupacionais (Bryant et al., 2006). Além disso, os pais podem estimular uma visão otimista de futuro e auxiliar os filhos na análise de forças pessoais, interesses e aspirações, assim como no exame do estilo de vida desejado e das oportunidades potenciais para atingir esse estilo de vida (Araújo et al., 2012).

Nesse sentido, os principais tipos de apoio evidenciados na literatura e que podem estar relacionados à tomada de decisões de carreira são o apoio emocional, o instrumental e o informacional (Araújo et al., 2012; Bryant et al., 2006; Dietrich, Kracke, \& Nurmi, 2011; Guan et al., 2015; Keller \& Whiston, 2008). O apoio emocional refere-se a comportamentos parentais de caráter afetivo que demonstram ao jovem preocupação com seu futuro e com as escolhas profissionais, e que transmitem a ideia de que o jovem é amado e estimado pelos seus pais (Pierce, Sarason, Sarason, Joseph, \& Henderson, 1996). Já o apoio instrumental são comportamentos que consistem em auxílios concretos na resolução de um determinado problema (Pierce et al., 1996), como ajuda financeira para cursar uma graduação ou para fazer um cursinho pré-vestibular. $\mathrm{O}$ apoio informacional consiste nas informações e orientações que o jovem recebe (Langford, Bowsher, Maloney, \& Lillis, 1997), como informações sobre o mercado de trabalho e as atividades profissionais.

Desse modo, é possível identificar que a influência parental pode envolver tanto aspectos objetivos e práticos, como apoio financeiro dos pais, quanto também aspectos de ordem subjetiva. Estes últimos podem tratar-se de influências não intencionais, tais como aprovação ou reprovação das escolhas profissionais, expectativas de resultados e cobranças, além das influências dos estilos de interação familiar, por exemplo, os padrões de apego (Almeida \& Melo-Silva, 2011).

Estudos demonstram que o apego seguro está relacionado a importantes aspectos do desenvolvimento profissional, como o comportamento exploratório (Vignoli, Croity-Belz, Chapeland, de Fillipis, \& Garcia, 2005) e a indecisão de carreira (correlação negativa) (BraunsteinBercovitz, Benjamin, Asor, \& Lev, 2012; Vignoli, 2009). A curiosidade e a segurança para solicitar ajuda são elementos importantes no processo de exploração e tomada de decisão vocacional. Desse modo, é possível inferir que pais que garantam relações pautadas em uma base segura (ou relações com confiança e segurança) parecem proporcionar mais oportunidades de exploração do mundo e, em consequência, uma exploração vocacional mais ampla, na medida em que uma base emocional segura contribui para o desenvolvimento da autonomia do jovem (Gonçalves, 2008). Em especial, o apoio dos pais às escolhas dos filhos tem sido relacionado ao comportamento exploratório vocacional (Bardagi, Lassance, \&
Teixeira, 2012; Dietrich \& Kracke, 2009; Dietrich et al., 2011; Guan et al., 2015; Palos \& Drobot, 2010), à tomada de decisão de carreira, ao otimismo de carreira (Garcia et al., 2015), à satisfação com a vida (Kim, Ahn, \& Fouad, 2016) e à busca pelos objetivos de carreira (Dietrich \& Salmela-Aro, 2013). A tomada de decisão inclui o balanço das informações e das alternativas profissionais obtidas pelo indivíduo com o objetivo de envolver-se em uma ação na qual haja comprometimento (Phillips \& Blustein, 1994). A exploração vocacional, por sua vez, consiste em um processo de busca de informações sobre si mesmo (busca pelo autoconhecimento) e sobre informações profissionais, tanto de forma comportamental quanto reflexiva (Jordaan, 1963).

O apoio dos pais influencia na confiança do jovem em desempenhar as tarefas importantes relacionadas às tomadas de decisões de carreira (Garcia et al., 2015). A exploração é mais provável de ocorrer e de ser bem-sucedida se o adolescente perceber seus pais como disponíveis e responsivos, como figuras de quem podem receber um suporte contínuo (Myrick, Green, \& Crenshaw, 2014). De fato, o suporte emocional e a segurança que os pais transmitem ao estabelecer um vínculo positivo com os filhos influenciam na forma como os jovens enfrentarão os desafios vividos no processo de transição da escola para universidade (Myrick et al., 2014).

Jovens que entendem ter um relacionamento afetuoso com seus pais, além de perceber que são apoiados nas decisões, tendem a buscar ocupações com características semelhantes às de seus pais (Tziner, Loberman, Dekel, \& Sharoni, 2012). Por outro lado, relações intrusivas entre pais e filhos tendem a prejudicar o desenvolvimento vocacional dos filhos. Estas podem gerar impactos, como o desengajamento em atividades ligadas ao comportamento exploratório ou um baixo compromisso com as escolhas de carreira, o que contribui para maior confusão diante das possíveis tomadas de decisão (Berríos-Alisson, 2005).

Considerando a adolescência como uma etapa em que o jovem é demandado a tomar decisões frente ao seu futuro profissional, o apoio dos pais torna-se importante nesse processo, tendo em vista que ele influência nas decisões de carreira (Garcia et al., 2015) e no comportamento exploratório dos jovens (Bardagi et al., 2012). Mesmo que o adolescente busque de forma autônoma informações sobre alguma profissão, por exemplo, os pais podem auxiliá-lo na qualidade da informação. Além disso, podem fazer questionamentos que contribuam para o autoconhecimento do adolescente (exploração de si) e sobre a carreira desejada, ajudando-o na tomada de decisão. Desse modo, torna-se relevante avaliar o envolvimento dos pais na carreira dos filhos por meio da preocupação, do interesse e da confiança destes nas decisões dos jovens. Ainda, torna-se importante avaliar quais estímulos estão sendo oferecidos e qual a disponibilidade dos pais para ajudar os filhos em suas escolhas profissionais. 
Portanto, avaliar a relação entre o apoio oferecido pelos pais no processo de desenvolvimento vocacional e a influência deste na carreira dos filhos é importante para uma melhor compreensão do desenvolvimento de carreira em adolescentes e jovens. Diversas pesquisas têm abordado diferentes variáveis relacionadas à possível influência parental nas escolhas e desenvolvimento de carreira, tais como as expectativas dos pais em relação à carreira dos filhos (Wang \& Heppner, 2002), a presença de apoio percebido (Ali \& Saunders, 2006; Dietrich \& Kracke, 2009; Keller \& Whiston, 2008; Turner, AllimanBrissett, Lapan, Udipi, \& Ergun, 2003) e a congruência parental, definida como a compatibilidade de valores, aspirações e objetivos entre pais e filhos (Sawitri, Creed, \& Zimmer-Gembeck, 2012). Estudos também evidenciam que o apoio e o envolvimento materno e paterno contribuem de forma distinta na carreira dos filhos. Em geral, o apoio materno tem sido mais associado ao desenvolvimento de carreira dos filhos uma vez que, possivelmente, a mães conversam mais com os filhos a respeito das questões que influenciam as decisões de carreira (Araújo et al., 2012; Palos \& Drobot, 2010), ao mesmo tempo em que oferecem mais apoio emocional (Palos \& Drobot, 2010). Essa relação de maior proximidade com as mães influenciam as filhas nas decisões de carreira uma vez que tendem a escolher ocupações com características similares às escolhidas pelas mães (Tziner et al., 2012).

Observa-se, no entanto, que a avaliação da influência dos pais na carreira nem sempre é feita por meio de instrumentos específicos que procurem avaliar tal influência de uma maneira abrangente. Em alguns estudos, existem apenas algumas questões relacionadas à influência parental, por exemplo, intrusividade dos pais na escolha de carreira dos filhos (Gavazzi, Reese, \& Sabatelli, 1998), ou ainda atitudes dos pais em relação a interesses específicos, como a matemática (Mulhern \& Rae, 1998). Outros estudos avaliam expectativas dos pais não exclusivamente relacionadas à carreira dos filhos, mas também a outros aspectos, como a maturidade pessoal e os relacionamentos amorosos (Wang \& Heppner, 2002). Além disso, torna-se importante avaliar as diferenças percebidas pelo apoio materno e paterno, considerando as diferenças reportadas na literatura (Araújo et al., 2012; Palos \& Drobot, 2010; Tziner et al., 2012). Tendo em vista a escassez de instrumentos nacionais que avaliem o apoio parental, considerando o apoio do pai e da mãe separadamente, voltado às questões de carreira, teve-se como objetivo neste estudo construir uma medida para avaliar aspectos da influência parental na escolha profissional.

Considerando os estudos que demonstram que o apoio dos pais auxilia os jovens na tomada de decisão (Dietrich \& Salmela-Aro, 2013; Garcia et al., 2015; Tziner et al., 2012) e também no engajamento em atividades vocacionalmente relevantes relacionadas à exploração de si e do contexto profissional (comportamento exploratório) (Araújo et al., 2012; Bryant et al., 2006; Dietrich et al.,
2011; Gonçalves, 2008; Guan et al., 2015; Myrick et al., 2014) a Escala de Envolvimento Parental nas Decisões Vocacionais (EEDPV) foi desenvolvida. Em contrapartida ao apoio, relações intrusivas têm sido relacionadas de forma negativa ao envolvimento em comportamento exploratório e também à indecisão de carreira (BerríosAlisson, 2005; Fan, Cheung, Leong, \& Cheung, 2014; Gavazzi et al., 1998). Logo, os aspectos relativos ao apoio à tomada de decisão, apoio ao comportamento exploratório e a intrusividade dos pais frente ao processo de desenvolvimento vocacional dos filhos foram considerados aspectos relevantes a serem mensurados por meio do instrumento.

\section{Método}

\section{Elaboração do Instrumento}

A escala foi construída com base na literatura da área de Orientação Profissional (Araújo et al., 2012; Bryant et al., 2006; Bardagi et al., 2012; Cheng \& Yuen, 2012; Turner et al., 2003; Whiston \& Keller, 2004), tendo sido elaborados inicialmente 18 itens compondo as seguintes dimensões: Apoio à tomada de decisão vocacional (seis itens que se referem a atitudes ou comportamentos parentais que transmitem ao adolescente a ideia de que seus pais se interessam pelo processo de tomada de decisão vocacional, incentivando a autonomia e oferecendo apoio); Apoio à exploração vocacional (quatro itens que se referem a atitudes ou comportamentos parentais que visam estimular os filhos a obterem informações vocacionalmente relevantes ou procurarem recursos que ajudem na tomada de decisão vocacional); e Intrusividade na tomada de decisão vocacional (quatro itens que se referem a comportamentos parentais que visam de algum modo influenciar diretivamente a escolha profissional dos filhos, seja por imposição explícita ou promovendo sentimentos de culpa em relação à escolha). Após essa etapa, foi realizada uma avaliação por três juízes que julgaram a pertinência dos itens às dimensões postuladas, chegando-se a um total de 14 itens. Em seguida, foi realizado um estudo piloto com adolescentes em que os itens não apresentaram necessidade de novas adequações. A escala possui grade de resposta em formato do tipo Likert, de 1 a 4 , sendo 1 = Definitivamente não, 2 = Mais pra não do que sim, $3=$ Mais pra sim do que não e $4=$ Definitivamente sim. $\mathrm{O}$ respondente deve optar por uma dessas quatro respostas separadamente para "Mãe" e "Pai" (ou figuras equivalentes indicadas pelos próprios respondentes), havendo espaços distintos para cada um.

Para este estudo, foi aplicada a escala de Escala de Envolvimento Parental na Decisão Vocacional (EEDPV), um questionário de caracterização dos participantes (buscou obter informações demográficas e caracterizar a amostra, como idade, sexo, nível de escolaridade e profissão dos pais, e aspectos socioeconômicos) e também a Escala de Maturidade para a Escolha Profissional - EMEP 
(Neiva, 2014). Essa escala avalia os níveis de maturidade profissional. É uma escala Likert, composta por 45 itens e divididos em cinco subescalas (determinação, responsabilidade, independência, autoconhecimento e conhecimento da realidade socioprofissional), apresentando índices adequados de consistência interna (variando de $\alpha=0,77$ a $\alpha=0,91$; Neiva, 1998).

\section{Participantes}

Participaram 390 jovens com idades entre 14 e 20 anos $(M=16,28 ; D P=1,15)$, residentes na cidade de Porto Alegre e região metropolitana do estado do Rio Grande do Sul; 51,8\% eram do sexo masculino e 48,2 do sexo feminino; $73,6 \%$ eram estudantes do ensino médio de escolas públicas e $26,4 \%$ eram de escolas particulares; $23,3 \%$ estavam cursando o primeiro ano, $48,5 \%$ o segundo ano e $28,2 \%$ o terceiro ano do ensino médio. Dados a respeito da escolaridade dos pais podem ser visualizados na Tabela 1. Em termos de situação profissional, no momento da pesquisa, $82,1 \%$ dos pais estavam trabalhando; $5,6 \%$ estava desempregado, $0,4 \%$ nunca trabalhou; $0,4 \%$ estava aposentado e $11,8 \%$ dos jovens não responderam. Em relação à situação profissional da mãe, 76,7\% estava trabalhando; $15,1 \%$ estava desempregada; $0,5 \%$ estava aposentada; $1,5 \%$ nunca trabalhou e $6,2 \%$ dos jovens não responderam.

Tabela 1

Nível de Escolaridade dos Pais

\begin{tabular}{|c|c|c|}
\hline Escolaridade & Pai & Mãe \\
\hline Ensino fundamental incompleto & $11,5 \%$ & $10,8 \%$ \\
\hline Ensino fundamental completo & $6,2 \%$ & $6,7 \%$ \\
\hline Ensino médio incompleto & $6,2 \%$ & $5,9 \%$ \\
\hline Ensino médio completo & $19,2 \%$ & $22,3 \%$ \\
\hline Ensino superior incompleto & $11 \%$ & $12,6 \%$ \\
\hline Ensino superior completo & 25,9 & $25,1 \%$ \\
\hline Pós-graduação & $12,6 \%$ & $14,6 \%$ \\
\hline Não respondeu & $7,4 \%$ & $2,1 \%$ \\
\hline
\end{tabular}

\section{Procedimentos e Considerações Éticas}

A pesquisa foi aprovada pelo Comitê de Ética em Pesquisa do Instituto de Psicologia da UFRGS (protocolo 15095613.0.0000.5334). As escolas foram selecionadas por conveniência, tendo autorizado a participação por meio do Termo de Concordância Institucional. A aplicação dos questionários foi feita em sala de aula, de maneira coletiva, tendo duração máxima de 30 minutos. Os adolescentes que aceitaram participar da pesquisa assinaram o Termo de Assentimento e seus responsáveis, o Termo de Consentimento Livre e Esclarecido.

\section{Análise dos Dados}

Foram realizadas análises fatoriais exploratórias com o método Minimum Rank Factor Analysis (MRFA, Shapiro \& Ten Berge, 2002; Ten Berge \& Kiers, 1991) com rotação oblíqua Direct Oblimin, conduzidas pelo software Factor 10.8 (Lorenzo-Seva \& Ferrando, 2006; Lorenzo-Seva \& Ferrando, 2013), esperando-se encontrar os três fatores inicialmente elaborados. Empregou-se o método de Hull (Lorenzo-Seva, Timmerman, \& Kiers, 2011) para seleção dos fatores. Uma vez que os itens são medidos em uma escala ordinal de quatro pontos, optou-se pela fatoração de matriz de correlações policóricas por ser considerada estimativa de correlação mais adequadas para esse tipo de medida. A fidedignidade foi verificada por meio do alfa de Cronbach.

\section{Resultados}

Inicialmente, verificou-se a adequação da matriz correlacional para a análise fatorial. Os testes de KaiserMeyer-Olkin (KMO) e de Esfericidade de Bartlett foram adotados para avaliar a adequação dos dados para a análise fatorial. Os resultados indicaram adequação dos dados para esse tipo de análise $(\mathrm{KMO}=0,85$ e o teste de esfericidade de Bartlett foi significativo; $p<0,001$ ). Para essa análise, foram extraídos três fatores (explicando $61,32 \%$ da variância total), conforme pode ser observado na Tabela 2. Contudo, o método de Hull indicou a extração de dois fatores.

Nessa análise, observou-se que o item 9, correspondente à dimensão intrusividade na tomada de decisão vocacional, apresentou carga cruzada com a de apoio à tomada de decisão vocacional; o item 1, que pertencia à dimensão intrusividade na tomada de decisão vocacional, carregou na dimensão apoio à tomada de decisão vocacional. Além disso, os itens 10 e 12 pertencentes inicialmente à dimensão apoio à tomada de decisão vocacional apresentaram carga cruzada com a de apoio à exploração vocacional.

Desse modo, nova análise foi realizada, extraindo-se dois fatores. Uma vez que alguns itens apresentaram cargas cruzadas, identificou-se, a partir de critérios teóricos, que a dimensão instrusividade pode ser uma 
questão pontual inserida na falta de apoio, especialmente relacionada ao apoio à tomada de decisão vocacional. Desse modo, optou-se por retirar os itens da dimensão intrusividade, mantendo apenas o item 9, por ser um item que reflete a ausência de apoio à tomada de decisão vocacional.

Tabela 2

Estrutura Fatorial dos Itens da EEDPV

\begin{tabular}{|c|c|c|c|}
\hline \multirow{2}{*}{ Itens } & \multicolumn{3}{|c|}{ Fatores } \\
\hline & 1 & 2 & 3 \\
\hline $\begin{array}{l}\text { 4. Minha mãe/meu pai pensa que eu devo escolher uma determinada } \\
\text { profissão e me pressiona para que eu a escolha. }\end{array}$ & 0,57 & $-0,27$ & 0,06 \\
\hline $\begin{array}{l}\text { 9. Eu sinto que minha mãe/meu pai ficaria desapontada(o) se eu } \\
\text { não escolhesse a profissão que ela/ele deseja para mim. }\end{array}$ & 0,51 & $-0,45$ & 0,05 \\
\hline $\begin{array}{l}\text { 13. Minha mãe/meu pai tenta impor suas ideias a respeito do que devo estudar } \\
\text { ou no que devo trabalhar no futuro. }\end{array}$ & 0,89 & 0,06 & $-0,05$ \\
\hline $\begin{array}{l}\text { 1. Minha mãe/meu pai fala mal das profissões ou dos tipos de trabalho } \\
\text { que eu venho pensando para mim. }\end{array}$ & 0,15 & $-0,66$ & 0,18 \\
\hline $\begin{array}{l}\text { 3. Minha mãe/meu pai apoia minhas decisões relacionadas aos meus } \\
\text { estudos no futuro ou ao trabalho que quero ter no futuro. }\end{array}$ & 0,08 & 0,75 & 0,07 \\
\hline 5. Minha mãe/meu pai aceita que eu escolha a profissão ou trabalho que eu queira. & $-0,08$ & 0,79 & $-0,10$ \\
\hline $\begin{array}{l}\text { 6. Minha mãe/meu pai mostra interesse quando eu digo a ela/ele que } \\
\text { estou pensando em seguir uma determinada profissão ou tipo de trabalho. }\end{array}$ & 0,03 & 0,66 & 0,25 \\
\hline $\begin{array}{l}\text { 10. Eu sei que posso contar com minha mãe/meu pai caso eu precise de ajuda } \\
\text { para poder tomar decisões sobre o meu futuro profissional. }\end{array}$ & $-0,06$ & 0,50 & 0,35 \\
\hline $\begin{array}{l}\text { 12. Eu percebo que minha mãe/meu pai confia que eu sou capaz de tomar } \\
\text { boas decisões sobre que cursos fazer ou que trabalho escolher. }\end{array}$ & $-0,10$ & 0,53 & 0,31 \\
\hline $\begin{array}{l}\text { 2. Minha mãe/meu pai me encoraja a buscar informações sobre as diversas } \\
\text { possibilidades educacionais, de trabalho e de carreira que existem. }\end{array}$ & 0,06 & 0,22 & 0,47 \\
\hline $\begin{array}{l}\text { 7. Minha mãe/meu pai me ajuda a procurar materiais informativos } \\
\text { sobre as diferentes profissões, cursos técnicos ou faculdades. }\end{array}$ & 0,14 & 0,12 & 0,60 \\
\hline $\begin{array}{l}\text { 8. Minha mãe/meu pai procura saber o que eu penso sobre o meu futuro } \\
\text { em termos de estudo ou trabalho. }\end{array}$ & 0,07 & 0,24 & 0,65 \\
\hline $\begin{array}{l}\text { 11. Minha mãe/meu pai me estimula a procurar orientação sobre cursos } \\
\text { e profissões em feiras de profissões ou serviços de orientação profissional. }\end{array}$ & 0,19 & 0,24 & 0,48 \\
\hline $\begin{array}{l}\text { 14. Minha mãe/meu pai conversa comigo sobre os planos que eu tenho } \\
\text { para a minha vida profissional ou de trabalho. }\end{array}$ & $-0,09$ & $-0,12$ & 0,94 \\
\hline
\end{tabular}

Nota. 1 - itens relativos à intrusividade; 2 - itens relativos ao apoio à decisão; 3 - itens relativos ao apoio à exploração

Assim, foi realizada nova análise, com 11 itens. Os resultados indicaram adequação dos dados para a análise fatorial $(\mathrm{KMO}=0,86 \mathrm{e}$ o teste de esfericidade de Bartlett foi significativo; $p<0,001)$. Para esta análise, foram extraídos dois fatores a partir da indicação do método de Hull, explicando $58,80 \%$ da variância total. Os componentes e as cargas podem ser visualizados na Tabela 3. É possível identificar que o fator 1 corresponde ao Apoio à tomada de decisão vocacional e o fator 2 ao Apoio à exploração vocacional. O item 8 , na primeira análise com três fatores, pertencia ao fator referente à tomada de decisão. Contudo, o fato deste item apresentar conteúdo que se refere a empregar comportamentos de troca de informações entre pais e filhos parece estar mais próximo à dimensão comportamento exploratório dos filhos estando, desse modo, vinculado a essa dimensão. 
Tabela 3

Estrutura Fatorial dos Itens da EEDPV

\begin{tabular}{|c|c|c|}
\hline \multirow{2}{*}{ Itens } & \multicolumn{2}{|c|}{ Fatores } \\
\hline & 1 & 2 \\
\hline $\begin{array}{l}\text { 3. Minha mãe/meu pai apoia minhas decisões relacionadas aos meus estudos } \\
\text { no futuro ou ao trabalho que quero ter no futuro. }\end{array}$ & 0,65 & 0,13 \\
\hline 5. Minha mãe/meu pai aceita que eu escolha a profissão ou trabalho que eu queira. & 0,83 & $-0,14$ \\
\hline $\begin{array}{l}\text { 6. Minha mãe/meu pai mostra interesse quando eu digo a ela/ele que } \\
\text { estou pensando em seguir uma determinada profissão ou tipo de trabalho. }\end{array}$ & 0,61 & 0,27 \\
\hline $\begin{array}{l}\text { 9. Eu sinto que minha mãe/meu pai ficaria desapontada(o) se eu não } \\
\text { escolhesse a profissão que ela/ele deseja para mim. }\end{array}$ & $-0,70$ & 0,24 \\
\hline $\begin{array}{l}\text { 10. Eu sei que posso contar com minha mãe/meu pai caso eu precise } \\
\text { de ajuda para poder tomar decisões sobre o meu futuro profissional. }\end{array}$ & 0,56 & $0,30^{*}$ \\
\hline $\begin{array}{l}\text { 12. Eu percebo que minha mãe/meu pai confia que eu sou capaz de tomar } \\
\text { boas decisões sobre que cursos fazer ou que trabalho escolher. }\end{array}$ & 0,63 & 0,24 \\
\hline $\begin{array}{l}\text { 2. Minha mãe/meu pai me encoraja a buscar informações sobre as diversas } \\
\text { possibilidades educacionais, de trabalho e de carreira que existem. }\end{array}$ & 0,18 & 0,49 \\
\hline $\begin{array}{l}\text { 7. Minha mãe/meu pai me ajuda a procurar materiais informativos } \\
\text { sobre as diferentes profissões, cursos técnicos ou faculdades. }\end{array}$ & $-0,02$ & 0,70 \\
\hline $\begin{array}{l}\text { 8. Minha mãe/meu pai procura saber o que eu penso sobre o meu futuro } \\
\text { em termos de estudo ou trabalho. }\end{array}$ & 0,21 & 0,66 \\
\hline $\begin{array}{l}\text { 11. Minha mãe/meu pai me estimula a procurar orientação sobre cursos e } \\
\text { profissões em feiras de profissões ou serviços de orientação profissional. }\end{array}$ & 0,07 & 0,60 \\
\hline $\begin{array}{l}\text { 14. Minha mãe/meu pai conversa comigo sobre os planos que eu tenho } \\
\text { para a minha vida profissional ou de trabalho. }\end{array}$ & $-0,08$ & 0,88 \\
\hline
\end{tabular}

Nota. *valor ajustado - valor exato: 0,297

A consistência interna foi investigada por meio do alfa de Cronbach, averiguando-se os valores para pai e mãe separadamente e também para o casal (para essa medida foi calculada a média dos escores da mãe e do pai). Tais dados podem ser visualizados na Tabela 4 .

Com o intuito de analisar a relação entre a EEDPV e variáveis teoricamente correlatas, foi realizada análise de correlação de Pearson entre a escala e a EMEP. Foi analisada a EEDPV com o escore combinado, sendo encontradas correlações estatisticamente significativas entre a dimensão apoio à tomada de decisão vocacional e responsabilidade $(r=0,19 ; p=0,05)$, determinação $(r=0,14$; $p=0,04)$, conhecimento da realidade $(r=0,19 ; p=0,004)$, autoconhecimento $(r=0,25 ; p<0,001)$, independência $(r=0,17 ; p=0,012)$ e EMEP total $(r=0,25 ; p<0,001)$. A dimensão apoio à exploração vocacional apresentou correlações estatisticamente significativas com as dimensões conhecimento da realidade $(r=0,25 ; p<0,001)$, autoconhecimento $(r=0,17 ; p<0,01)$, EMEP total $(r=0,15$; $p=0,023)$. A escala total EEDPV apresentou correlação estatisticamente significativa com as dimensões responsabilidade $(r=0,14 ; p=0,035)$, determinação $(r=0,14$; $p=0,04)$, conhecimento da realidade $(r=0,26 ; p<0,001)$, autoconhecimento $(r=0,24 ; p<0,001)$ e EMEP total $(\mathrm{r}=0,24 ; p<0,001)$.

Tabela 4

Índices de Consistência Interna da EEDPV

\begin{tabular}{lccc}
\hline & Pai & Mãe & Casal \\
\hline Apoio à tomada de decisão vocacional & 0,76 & 0,76 & 0,77 \\
Apoio à exploração vocacional & 0,82 & 0,76 & 0,76 \\
Escala total & 0,85 & 0,80 & 0,80 \\
\hline
\end{tabular}




\section{Discussão}

O objetivo deste estudo foi analisar as propriedades psicométricas da EEDPV. Esta tem por finalidade avaliar a percepção de adolescentes sobre o envolvimento parental no processo de escolha vocacional. A versão inicial foi composta por 14 itens, elaborados a partir da literatura da área de orientação profissional com as dimensões apoio à tomada de decisão vocacional, apoio à exploração vocacional e instrusividade na tomada de decisão vocacional. Contudo, o método utilizado para retenção dos fatores indicou a existência de dois fatores, o qual apresentou alguns itens com cargas cruzadas. Além disso, foi identificado que a dimensão intrusividade refere-se a um tipo de apoio pontual, ou melhor, inverso ao apoio à tomada de decisão vocacional uma vez que tem sido relacionado à dificuldade do jovem de tomar decisões profissionais (Fan et al., 2014). Assim, optou-se por deixar somente um item dessa dimensão que se referia à ausência de apoio à decisão. Desse modo, a escala foi composta por itens relativos ao apoio os pais na carreira dos filhos no que se refere à decisão profissional e ao comportamento exploratório.

Em virtude dessa relação, foi realizada correlação entre a EEDPV e uma escala que avalia maturidade para a escolha profissional. De modo geral, embora tenham sido encontrados índices de correlação baixos, eles foram significativos evidenciando que a percepção de apoio parental apresenta relação com a maturidade de carreira (Bae, 2017; Lee, Lee, Song, \& Kim, 2015; Whiston \& Keller, 2004). Além disso, foram evidenciados índices de confiabilidade robustos, tanto para a percepção do filho frente ao apoio de cada um dos pais quanto para o casal, confirmando a importância do apoio dos pais nas tomadas de decisão de carreira e no comportamento exploratório dos filhos (Araújo et al., 2012; Dietrich \& Salmela-Aro, 2013; Garcia et al., 2015; Guan et al., 2015; Myrick et al., 2014; Tziner et al., 2012). Considerando esse aspecto, sugerem-se para uso futuro da escala que possam ser consideradas as respostas de cada um dos pais, a depender do objetivo do estudo.

Essa escala pode ser utilizada na prática de aconselhamento de carreira de adolescentes, visto que facilita o acesso do orientador às questões relacionadas à influência parental no processo de escolha profissional. Nesse contexto, pode ser utilizada para contribuir com o processo de autoconhecimento dos adolescentes, à medida que eles podem refletir sobre a influência que seus pais exercem nos movimentos de exploração de si e do mundo, bem como nas decisões profissionais que tomam. Compreender os possíveis antecedentes da indecisão de carreira tem se mostrado importante para subsidiar intervenções junto aos clientes de aconselhamento de carreira (Downing \& Nauta, 2010). Destaca-se o fato de que esse instrumento permite diferenciar o papel que cada um dos pais desempenha no processo de escolha vocacional, conforme a percepção do adolescente. Assim, pode-se obter maior precisão com relação à realidade vivenciada pelo adolescente, havendo mais subsídios para possíveis intervenções junto a ele e aos próprios pais, conforme tem sido recomendado na literatura (Almeida \& MeloSilva, 2011; Whiston \& Keller, 2004).

No contexto da pesquisa, sugere-se que seja relacionada com variáveis relevantes para o desenvolvimento de carreira, tais como adaptabilidade de carreira, comportamento exploratório, autoeficácia para decisão de carreira, com o objetivo de verificar os possíveis efeitos da influência parental sobre elas. Pelo que indica a literatura, é provável que desfechos positivos estejam relacionados à percepção de apoio parental, como a autonomia (Gordon \& Cui, 2015), a autoeficácia para a tomada de decisão profissional (Fouad, Kim, Ghosh, Chang, \& Figueiredo, 2016; Ginevra, Nota, \& Ferrari, 2013; Sawitri, Creed, \& Zimmer-Gembeck, 2015; Sovet \& Metz, 2014), satisfação com a carreira (Gordon \& Cui, 2015) e o comportamento exploratório (Bardagi et al., 2012; Sawitri et al., 2015). Tais aspectos podem ser investigados em futuros estudos, os quais são recomendados também para que se possam obter mais evidências de validade do instrumento.

Enfim, este estudo desenvolveu uma escala inédita no campo da orientação profissional no contexto brasileiro, com evidências adequadas de validade e fidedignidade. Recomenda-se, para pesquisas futuras, a ampliação e diversificação da amostra, por exemplo, com universitários. Estudos demonstram que há influência da família em variáveis de carreira também nesse público, como comportamento exploratório, identidade vocacional, tomada de decisão (Braunstein-Bercovitz et al., 2012; Guan et al., 2015; Kim et al., 2016; Mojgan, Abdul Kadir, Noah, \& Hassan, 2013; Tate et al., 2015; Whiston \& Keller, 2004). Também é pertinente realizar correlações com outros instrumentos, a fim de se reforçar as evidências de validade dela. A escala demonstra potencial para uso em pesquisas e na prática de orientação profissional.

\section{Referências}

Ali, S., \& Saunders, J. (2006). College expectations of rural appalachian youth: An exploration of social cognitive career theory factors. The Career Development Quarterly, 55(1), 38-51. doi: 10.1002/j.2161-0045.2006.tb00003.x

Almeida, F., \& Melo-Silva, L. (2011). Influência dos pais no processo de escolha profissional dos filhos: uma revisão da literatura. Psico-USF, 16(1), 75-85. doi: 10.1590/S1413-82712011000100009 
Araújo, A., Taveira, M. C., Mota, A., Oliviera, I., Pinto, J., Viamonte, I., \& Faria, L. (2012). Parental participation in adolescents' educational and career experiences and planning: A study with portuguese parents. Em 6th International Technology, Education and Development Conference (pp. 3631-3637), Espanha.

Bae, S. (2017). An analysis of career maturity among Korean youths using latent growth modeling. School Psychology International, 38(4), 434449. doi: $10.1177 / 0143034317709527$

Bardagi, M. P., Lassance, M. C. P., \& Teixeira, M. A. P. (2012). O contexto familiar e o desenvolvimento vocacional de jovens. Em M. N. Baptista \& M. L. M. Teodoro (Eds.). Psicologia de família: Teoria, avaliação e intervenções (pp. 135-144). Porto Alegre: Artmed.

Berríos-Alisson, A. (2005). Family influences on college students' occupational identity. Journal of Career Assessment, 13(2), 233-247. doi: $10.1177 / 1069072704270320$

Braunstein-Bercovitz, H., Benjamin, B., Asor, S., \& Lev, M. (2012). Insecure attachment and career indecision: Mediating effects of anxiety and pessimism. Journal of Vocational Behavior, 81(2), 236-244. doi: 10.1016/j.jvb.2012.07.009

Bryant, B., Zvonkovic, A., \& Reynolds, P. (2006). Parenting in relation to child and adolescent vocational development. Journal of Vocational Behavior, 69(1), 149-175. doi: 10.1016/j.jvb.2006.02.004

Cheng, S., \& Yuen, M. (2012). Validation of the career-related parent support scale among chinese high school students. The Career Development Quarterly, 60(4), 367-374. doi: 10.1002/j.2161-0045.2012.00028.x

Dietrich, J., \& Kracke, B. (2009). Career-specific parental behaviors in adolescents' development. Journal of Vocational Behavior, 75(2), 109119. doi: 10.1016/j.jvb.2009.03.005

Dietrich, J., \& Kracke, B., \& Nurmi, J. (2011). Parents' role in adolescents' decision on college major: A weekly diary study. Journal of Vocational Behavior, 79(1), 134-144. doi: 10.1016/j.jvb.2010.12.003

Dietrich, J., \& Salmela-Aro, K. (2013). Parental involvement and adolescents' career goal Đursuit during the post-school transition. Journal of Adolescence, 36(1), 121-128. doi: 10.1016/j.adolescence.2012.10.009

Downing, H., \& Nauta, M. (2010). Separation-individuation, exploration, and identity diffusion as mediators of the relationship between attachment and career indecision. Journal of Career Development, 36(3), 207-227. doi: 10.1177/0894845309345848

Fan, W., Cheung, F., Leong, F., \& Cheung, S. (2014). Contributions of family factors to career readiness: A cross-cultural comparison. The Career Development Quarterly, 62(3), 194-209. doi: 10.1002/j.2161-0045.2014.00079.x

Fouad, N., Kim, S., Ghosh, A., Chang, W., \& Figueiredo, C. (2016). Family Influence on Career Decision Making: Validation in India and the United States. Journal of Career Assessment, 24(1), 197-212. doi: 10.1177/1069072714565782

Garcia, P., Restubog, S., Bordia, P., Bordia, S., \& Roxas, R. (2015). Career optimism: The roles of contextual support and career decisionmaking self-efficacy. Journal of Vocational Behavior, 88, 10-18. doi: 10.1016/j.jvb.2015.02.004

Gavazzi, S., Reese, M., \& Sabatelli, R. (1998). Conceptual development and empirical use of the family intrusiveness scale. Journal of Family Issues, 19(1), 65-74. doi: 10.1177/019251398019001005

Ginevra, M., Nota, L., \& Ferrari, L. (2013). Parental support in adolescents' career development: Parents' and children's perceptions. The Career Development Quarterly, 63(1), 1-15. doi: 10.1002/j.2161-0045.2015.00091.x

Gonçalves, C. M. (2008). Pais aflitos, filhos com futuro incerto? Um estudo sobre a influência das famílias na orientação vocacional dos jovens. Lisboa, Portugal: Edições Fundação Calouste Gulbenkian.

Gordon, M., \& Cui, M. (2015). Positive parenting during adolescence and career success in young adulthood. Journal of Child and Family Studies, 24(3), 762-771. doi: 10.1007/s10826-013-9887-y

Guan, Y., Wang, F., Liu, H., Ji, Y., Jia, X., Fang, Z., ... Li, C. (2015). Career-specific parental behaviors, career exploration and career adaptability: A three-wave investigation among Chinese undergraduates. Journal of Vocational Behavior, 86, 95-103. doi: 10.1016/j. jvb.2014.10.007

Keller, B., \& Whiston, S. (2008). The role of parental influences on young adolescents' career development. Journal of Career Assessment, 16(2), 198-207. doi: 10.1177/1069072707313206

Kim, S., Ahn, T., \& Fouad, N. (2016). Family influence on korean students' career decisions: A social cognitive perspective. Journal of Career Assessment, 24(3) 513-526. doi: 10.1177/1069072715599403

Jordaan, J. P. (1963). Exploratory behaviour: The formation of self and occupational concepts. Em D. E. Super, R. Starshevsky, R. Matlin, \& J. P. Jordan (Eds.) Career development: Self-concept theory. New York: College Entrance Board.

Langford, C., Bowsher, J., Maloney, J., \& Lillis, P. (1997). Social support: A conceptual analysis. Journal of Advanced Nursing, 25, 95-100. doi: 10.1046/j.1365-2648.1997.1997025095.x

Lee, S., Lee, H., Song, H., \& Kim, S. (2015). The relationship between attachment and career maturity: The mediating role of self-efficacy. International Social Work, 58(1) 153-164. 1 doi: 0.1177/0020872812456053

Lorenzo-Seva, U., \& Ferrando, P. J. (2006). FACTOR: A computer program to fit the exploratory factor analysis model. Behavior Research Methods, 38(1), 88-91.

Lorenzo-Seva, U., Timmerman, M. E., \& Kiers, H. A. (2011). The hull method For selecting the number of common Factors. Multivariate Behavioral Research, 46(2), 340-364. doi: 10.1080/00273171.2011.564527.

Lorenzo-Seva, U., \& Ferrando, P.J. (2013). FACTOR 9.2: A comprehensive program for fitting exploratory and semiconfirmatory factor analysis and IRT Models. Applied Psychological Measurement, 37(6), 497-498. doi: 10.1177/0146621613487794

Myrick, A., Green, E., \& Crenshaw, D. (2014). The influence of divergent parental attachment styles on adolescent maturation: Implications for family counseling practitioners. The Family Journal: Counseling and Therapy for Couples and Families, 22(1), 35-42. doi: $10.1177 / 1066480713491217$

Mojgan, F., Abdul Kadir, R., Noah, S., \& Hassan. (2013). The relation of career indecision and parental attachment among iranian undergraduate students. International Journal for the Advancement of Counselling, 35(4), 251-260. doi: 10.1007/s10447-012-9180-6

Mulhern, F., \& Rae, R. (1998). Development of a shortened form of the fennema-sherman mathematics aptitudes scales. Educational and Psychological Measurement, 58(2), 295-306. doi: 10.1177/0013164498058002012

Neiva, K. M. C. (1998). Escala de maturidade para a escolha profissional (EMEP): Estudo de validade e fidedignidade. Revista Unib, 6, 43-61.

Neiva, K. M. C. (2014). Escala de maturidade para a escolha profissional (EMEP). São Paulo: Vetor

Palos, R., \& Drobot, L. (2010). The impact of family influence on the career choice of adolescents. Procedia Social and Behavioral Sciences, 2(2), 3407-3411. doi: 10.1016/j.sbspro.2010.03.524 
Phillips, S., \& Blustein, D. L. (1994). Readiness for career choices: Planning, exploring and deciding. The Career Development Quarterly, 43(1), 63-69. doi: 10.1002/j.2161-0045.1994.tb00847.x

Pierce, G., Sarason, B., Sarason, I., Joseph, H., \& Henderson, C. (1996). Conceptualizing and assessing social support in the context of the family. Em G. Pierce, B. Sarason, \& I. Sarason (Eds.). Handbook of social support and the family (pp. 03-24). New York: Plenum Press.

Sawitri, D., Creed, P., \& Zimmer-Gembeck, M. (2012). The adolescent-parent career congruence scale: Development and initial validation. Journal of Career Assessment, 21(2), 210-226. doi: 10.1177/1069072712466723

Sawitri, D., Creed, P., \& Zimmer-Gembeck, M. (2015). Longitudinal relations of parental influences and adolescent career aspirations and actions in a collectivist society. Journal of Research on Adolescence, 25(3), 551-563. doi: 10.1111/jora.12145

Shapiro, A., \& Ten Berge, J. M. F. (2002). Statistical inference of minimum rank factor analysis. Psychometrika, 67(1), 79-94. doi: 10.1007/ BF02294710

Sovet, L., \& Metz, A. (2014). Parenting styles and career decision-making among French and Korean adolescents. Journal of Vocational Behavior, 84(3), 345-355. doi: 10.1016/j.jvb.2014.02.002

Tate, K., Fouad, N., Marks, L., Young, G., Guzman, E., \& Williams, E. (2015). Underrepresented first-generation, low-income college students' pursuit of a graduate education: Investigating the influence of self-efficacy, coping efficacy, and family influence. Journal of Career Assessment, 23(3) 427-444. doi: 10.1177/1069072714547498

Ten Berge, J. M. F., \& Kiers, K., A., L. (1991). A numerical approach to the approximate and the exact minimum rank of a covariance matrix. Psychometrika, 56(2), 309-315. doi: 10.1007/BF02294464

Turner, S., Alliman-Brissett, A., Lapan, R., Udipi, S., \& Ergun, D. (2003). The career related parent support scale. Measurement and Evaluation in Counseling and Development, 36(2), 86-94. doi: 10.1037/t41110-000

Tziner, A., Loberman, G., Dekel, Z., \& Sharoni, G. (2012). The influence of the parent offspring relationship on young people's career preferences. Revista de Psicología del Trabajo y de las Organizaciones, 28(2), 99-105. doi: 10.5093/tr2012a8

Vignoli, E. (2009). Inter-relationships among attachment to mother and father, self-esteem, and career indecision. Journal of Vocational Behavior, 75(2), 91-99. doi: 10.1016/j.jvb.2009.04.007

Vignoli, E., Croity-Belz, S., Chapeland, V., de Fillipis, A., \& Garcia, M. (2005). Career exploration in adolescents: The role of anxiety, attachment, and parenting style. Journal of Vocational Behavior, 67(2), 153-168. doi: 10.1016/j.jvb.2004.08.006

Wang, L., \& Heppner, P. (2002). Assessing the impact of parental expectations and psychological distress on Taiwanese college students. The Counseling Psychologist, 30(4), 582-608. doi: 10.1177/00100002030004006

Whiston, S., \& Keller, B. (2004). The influences of the family of origin on career development: A review and analysis. The Counseling Psychologist, 32(4), 493-568. doi: 10.1177/0011000004265660

\section{Sobre os autores}

Cássia Ferrazza Alves é Doutora em Psicologia/UFRGS e professora de Psicologia/FSG - Centro Universitário.

Luciana Rubensan Ourique Masiero é doutora em Psicologia/UFRGS e psicóloga Organizacional/PROCERGS.

Ana Cristina Garcia Dias é Pós-Doutora em Psicologia/UFRGS, doutora em Psicologia USP/SP, professora do Departamento de Psicologia do Desenvolvimento e personalidade do Programa de Pós-Graduação em Psicologia/UFRGS e bolsista CNPq PQ2.

Marco Antônio P. Teixeira é Doutor em Psicologia/UFRGS e professor no Instituto de Psicologia/UFRGS e do Programa de PósGraduação em Psicologia/UFRGS. 